Check for updates

Cite this: RSC Adv., 2019, 9, 32258

Received 9th August 2019

Accepted 20th September 2019

DOI: 10.1039/c9ra06191f

rsc.li/rsc-advances

\section{Facile synthesis of mesoporous carbon microspheres/graphene composites in situ for application in supercapacitors}

\author{
Jing Chen, $\uparrow^{a}$ Youliang Cheng, $\dagger^{a}$ Qingling Zhang, ${ }^{a}$ Changqing Fang, (D) *a Linlin $W u^{a}$ \\ Mengsha $\mathrm{Bai}^{\mathrm{a}}$ and Yongtao $\mathrm{Yao}^{\mathrm{b}}$
}

Mesoporous carbon/graphene composites (MCG) have exhibited good electrochemical performances; however, the fixed mesoporous carbon, the low specific surface area, and porosity are the main obstacles in their application in supercapacitors. In this paper, mesoporous carbon microspheres/ graphene composites (MCMG) were synthesized in situ via a soft template method and subsequent thermal reduction by using cetyltrimethylammonium bromide (CTAB) as the structure-directing agent, and aqueous mesophase pitch (AMP) and graphene oxide (GO) as the carbon sources. The strong electrostatic interaction between GO/CTAB and AMP promoted the self-assembly of CTAB and AMP to form the MCMG precursor. The results showed that the CTAB concentration and aging temperature have an important effect on the morphology and pore structure of the synthesized MCMG. The high aging temperature promoted the formation of mesoporous carbon spheres and its diameter increased with the increase in the concentration of CTAB. The as-prepared MCMG at the aging temperature of $140{ }^{\circ} \mathrm{C}$ had obvious spherical and layered carbon materials after carbonization at $900{ }^{\circ} \mathrm{C}$. When the concentration of CTAB was $10.6 \mathrm{~g} \mathrm{~L}^{-1}$, the formed mesoporous carbon spheres with the diameter of 30-40 nm were uniformly dispersed among the layered graphenes in MCMG-140-0.2 (the aging temperature of $140{ }^{\circ} \mathrm{C}$ and the CTAB content of $0.2 \mathrm{~g}$ ). In addition, its specific surface area was 1150.5 $\mathrm{m}^{2} \mathrm{~g}^{-1}$ and the mesopore size was centered at $4.3 \mathrm{~nm}, 7.9 \mathrm{~nm}$, and $17.1 \mathrm{~nm}$. Compared with the MCMG precursor, the ordered degree of the mesopores for MCMG was reduced due to the high temperature carbonization. Importantly, the specific capacitance of MCMG-140-0.2 at the current density of $0.1 \mathrm{~A} \mathrm{~g}^{-1}$ was as high as $356.3 \mathrm{~F} \mathrm{~g}^{-1}$. Moreover, the specific capacitance of MCMG-140-0.2 at $1 \mathrm{~A} \mathrm{~g}^{-1}$ remained at $278.5 \mathrm{~F} \mathrm{~g}^{-1}$, the capacitance retention was $92.1 \%$ after 6000 cycles, and the coulombic efficiency was over $98 \%$ at a high current density of $2 \mathrm{~A} \mathrm{~g}^{-1}$. Therefore, the as-prepared MCMG can be an excellent candidate for electrode materials in supercapacitors.

\section{Introduction}

Supercapacitors are one of the most promising energy storage devices, which mainly include carbon-based double-layer capacitors and metal-based pseudocapacitors. ${ }^{1}$ For the former, many researchers have focused on developing highperformance graphene-based composites as electrode materials by inserting nanoparticles (such as $\mathrm{MnO}_{2}$ nanowires, ${ }^{2}$ $\mathrm{RuO}_{2}$ nanoparticles, ${ }^{3} \quad \mathrm{Fe}_{3} \mathrm{O}_{4}$ nanoparticles, ${ }^{4} \mathrm{Co}_{3} \mathrm{O}_{4}$ nanoparticles, ${ }^{5}$ carbon black, ${ }^{6}$ carbon nanotubes, ${ }^{7}$ and fullerenes ${ }^{8}$ ) into the sheets of graphene. In addition, N-doping of graphene sheets also is an effect strategy for improving its

${ }^{a} X i$ 'an University of Technology, Xi'an 710048, P. R. China.E-mail: fcqxaut@163.com; Fax: +8629 82312512; Tel: +862982312038

${ }^{b}$ National Key Laboratory of Science and Technology on Advanced Composites in Special Environments, Harbin Institute of Technology, Harbin 150001, P. R. China

$\dagger$ These authors contribute equally to the article. electrochemical performance. ${ }^{9}$ Recently, mesoporous carbon microspheres/graphene composites (MCMG) consisting of graphene and mesoporous carbon microspheres (MCMs) have found extremely important applications in supercapacitors, ${ }^{\mathbf{1 0 , 1 1}}$ lithium ion batteries, ${ }^{12,13}$ fuel cells,${ }^{14}$ solar cells, ${ }^{15}$ biosensors, ${ }^{16}$ catalyst carriers, ${ }^{17}$ and adsorption separation technology. ${ }^{18}$ Furthermore, non-spherical mesoporous carbon/graphene composites (MCG) have also attracted researchers' attentions. Lei and co-workers prepared surface functionalized CMK-5 using polydiallyl dimethyl ammonium chloride and assembled them into the layers of oxide graphene (GO) to synthesize the CMK-5/graphene composite, which exhibited excellent electrochemical properties. ${ }^{19} \mathrm{Tu}$ and co-workers used a similar method to insert S-doped CMK-3 into graphene sheets. ${ }^{20}$ Due to the hydrophobic effect of S, the cycling stability of the CMK-3/ graphene composite was improved. Besides, MCG also can be obtained by directly carbonizing the mixture of polymer and GO. ${ }^{21,22}$ Some researchers have mixed oxidized petroleum pitch 
and coal tar pitch with GO, and then activated the mixture by $\mathrm{KOH}$ to prepare the graphene-based composites rich in mesopores. ${ }^{23,24}$ Moreover, mesoporous carbon materials derived from petroleum pitch and coal tar pitch also showed excellent electrochemical performances. ${ }^{25,26}$

Recently, Zhao and co-workers incorporated the functionalized mesoporous silica spheres (MSS) with GO and then fabricated 3D MCMG with a specific surface area as high as $1496 \mathrm{~m}^{2}$ $\mathrm{g}^{-1}$ by the CVD method using MSS/GO as the template. ${ }^{27}$ The MCG with disordered pores can be obtained by using cetyltrimethylammonium chloride (CTACl) or cetyltrimethylammonium bromide (CTAB) as the structure-directing agent and tetraethoxysilane (TEOS) as the silicon source. ${ }^{28,29}$ When mesoporous silica/GO composites obtained by the triblock copolymer $\mathrm{PEO}_{20} \mathrm{PPO}_{70} \mathrm{PEO}_{20}$ (P123) were used as the hard template, MCG with different pores and morphologies were prepared. ${ }^{30}$ In addition, MCG also can be constructed after synthesizing the silica/carbon/GO composites using the triblock copolymer $\mathrm{PEO}_{106} \mathrm{PPO}_{70} \mathrm{PEO}_{106}$ (F127) as the structure-directing agent. ${ }^{31}$ The above mentioned studies usually concern the removal of the silica template, which may destroy some pore channels.

Instead, the soft templates for synthesizing mesoporous carbon materials are easily removed during the subsequent carbonization process. Moreover, different mesoporous pores are feasibly achieved by changing the templates. Therefore, the soft template method has been applied in the synthesis of MCMG and MCG. ${ }^{32}$ Jin and co-workers prepared cylindrical MCG with the specific surface area of $400 \mathrm{~m}^{2} \mathrm{~g}^{-1}$ by using F127 as the template and phenolic resin as the carbon source, which can be applied in fuel batteries. ${ }^{33}$ The precursor was constructed by depending on the strong hydrogen bonds between F127, GO, and the phenolic resin, and then MCG with high specific surface area and excellent conductivity was obtained by subsequent thermal reduction. ${ }^{34}$ After stripping GO by an ultrasonic method, MCG with a specific surface area of $545.8 \mathrm{~m}^{2} \mathrm{~g}^{-1}$ was synthesized by the soft template method, exhibiting a specific capacitance of $242 \mathrm{~F} \mathrm{~g}^{-1}$ in $6 \mathrm{M} \mathrm{KOH}$ as the electrolyte. ${ }^{35}$

Due to the limitation of template concentration and the type of carbon source, mesoporous carbon in the composites synthesized via a soft template method showed flaky or irregular shapes in the previous reports. ${ }^{32-35}$ It was required to synthesize MCM or MSS before constructing MCMG, ${ }^{27,34,36}$ and then the morphology of mesoporous carbon and the mesoporous structure was quite difficult to control in situ. To resolve the above problems, the MCMG precursors were synthesized in situ using $\mathrm{CTAB}$ as the structure-directing agent and aqueous mesophase pitch (AMP) as the carbon source in the GO dispersion in this work. Then, MCMG were obtained by removing $\mathrm{CTAB}$ via subsequent high-temperature carbonization. The effects of aging temperature and CTAB concentration on the morphology and microstructure of the MCMG were investigated. When the aging temperature was $140{ }^{\circ} \mathrm{C}$ and the CTAB concentration was $10.6 \mathrm{~g} \mathrm{~L}^{-1}$, the as-prepared MCMG sample rich in mesopores had the specific surface area as high as $1150.5 \mathrm{~m}^{2} \mathrm{~g}^{-1}$ and the diameter of the formed mesoporous carbon spheres was in the range of $30-40 \mathrm{~nm}$. Furthermore, they exhibited excellent electrochemical performances when used as electrode materials in supercapacitors.

\section{Experimental}

\subsection{Materials}

Natural graphite was purchased from Sinopharm Chemical Reagent Co. Ltd. Coal tar pitch was purchased from Wuhan Iron and Steel Co. Ltd. Concentrated $\mathrm{H}_{2} \mathrm{SO}_{4}(98 \%)$, concentrated $\mathrm{HNO}_{3}$ (68\%), concentrated $\mathrm{HCl}(36 \%)$, and $\mathrm{NaOH}$ were purchased from Xi'an Chemical Reagent Factory. $\mathrm{KMnO}_{4}$, $\mathrm{NaNO}_{3}, \mathrm{H}_{2} \mathrm{O}_{2}(30 \%)$, CTAB, and $\mathrm{KBr}$ were purchased from Tianjin Chemical Reagent Co. Ltd. Deionized water was used throughout the experiments and all the chemicals were used without any further purification.

\subsection{Preparation of AMP}

AMP was prepared by oxidizing coal tar pitch, alkali redissolution, and subsequent acid precipitation process. ${ }^{37}$ Typically, $5 \mathrm{~g}$ of coal tar pitch was added into $100 \mathrm{~mL}$ of the mixed acid (the volume ratio of concentrated $\mathrm{HNO}_{3}$ and concentrated $\mathrm{H}_{2} \mathrm{SO}_{4}$ was $3: 7$ ) with stirring at $80^{\circ} \mathrm{C}$. After keeping for $1 \mathrm{~h}$ in a water bath, the obtained black product was poured into a $1000 \mathrm{~mL}$ beaker with $500 \mathrm{~mL}$ of deionized water and was left undisturbed for $6 \mathrm{~h}$. The supernatant was decanted and the filtrated materials were washed with deionized water until the filtrate became neutral. The obtained product was dissolved in $1 \mathrm{M} \mathrm{NaOH}$ solution so that the $\mathrm{pH}$ of the system was above 12 and stirred at $80{ }^{\circ} \mathrm{C}$ for $1 \mathrm{~h}$. Subsequently, the above solution was filtrated and then $1 \mathrm{M} \mathrm{HCl}$ was added dropwise into the filtrate until the $\mathrm{pH}$ of the solution was below 2. After leaving undisturbed for $12 \mathrm{~h}$, the precipitate was collected by centrifugation and washed with deionized water 3 times to remove the acid, followed by drying at $100{ }^{\circ} \mathrm{C}$ in a vacuum oven to obtain the AMP product.

\subsection{Synthesis of MCMG}

The GO used in this work was prepared by modified Hummer's method. ${ }^{38} \mathrm{~A}$ certain amount of GO was added into $\mathrm{NaOH}$ solution $(\mathrm{pH}=12)$ and sonicated for $1 \mathrm{~h}$ to obtain $0.6 \mathrm{mg} \mathrm{mL}^{-1} \mathrm{GO}$ solution. The appropriate amount of CTAB was added into the above GO solution with stirring until CTAB was completely dissolved to obtain GO/CTAB dispersion. Subsequently, $0.3 \mathrm{~g}$ of AMP was added into $190 \mathrm{~mL}$ of $\mathrm{GO} / \mathrm{CTAB}$ dispersion with stirring for $1 \mathrm{~h}$ at $30^{\circ} \mathrm{C}$ in a water bath. The above mixture was transferred to a $200 \mathrm{~mL}$ hydrothermal reactor with Teflon lining and then aged at the setting temperature for $48 \mathrm{~h}$. The obtained mixture was filtrated and washed with deionized water, then dried to get the MCMG precursor. The content of CTAB was set as $0.06 \mathrm{~g}$, $0.1 \mathrm{~g}, 0.15 \mathrm{~g}, 0.2 \mathrm{~g}, 0.24 \mathrm{~g}$, and $0.3 \mathrm{~g}$ (the concentration was $3.1 \mathrm{~g} \mathrm{~L}^{-1}, 5.3 \mathrm{~g} \mathrm{~L}^{-1}, 7.9 \mathrm{~g} \mathrm{~L}^{-1}, 10.6 \mathrm{~g} \mathrm{~L}^{-1}, 12.6 \mathrm{~g} \mathrm{~L}^{-1}$, and $15.8 \mathrm{~g}$ $\mathrm{L}^{-1}$, respectively). The mass ratio of AMP to CTAB was $5: 1$, $3: 1,2: 1,3: 2,5: 4$, and $1: 1$, respectively. The aging temperature was set as $130{ }^{\circ} \mathrm{C}$ and $140{ }^{\circ} \mathrm{C}$, respectively. The carbonization process of the MCMG precursors under $\mathrm{N}_{2}$ 
atmosphere was as follows: the heating rate was set as $1{ }^{\circ} \mathrm{C} \mathrm{min^{-1 }}$ in the range of $25-220{ }^{\circ} \mathrm{C}, 2{ }^{\circ} \mathrm{C} \mathrm{min}^{-1}$ in the range of $220-450{ }^{\circ} \mathrm{C}$, and $3{ }^{\circ} \mathrm{C} \mathrm{min}^{-1}$ in the range of $450-900{ }^{\circ} \mathrm{C}$. In addition, the soaking time at $450{ }^{\circ} \mathrm{C}$ and $900{ }^{\circ} \mathrm{C}$ was $2 \mathrm{~h}$. Finally, the reaction system was naturally cooled to room temperature and MCMG was obtained. The as-prepared MCMG samples under the conditions of different CTAB concentrations and aging temperatures were labeled as MCMG- $x-y$, where " $x$ " was the aging temperature and " $y$ " was the CTAB mass.

\subsection{Characterization}

The morphology and structure of samples were investigated by high resolution transmission electron microscopy (TEM, JEOL3010) and scanning electron microscopy (SEM, SU-8000). Small angle X-ray diffraction (SAXRD) patterns were obtained by using an X-ray diffractometer (XRD Shimadzu Limited XRD-700) with a $\mathrm{Cu} \mathrm{K} \alpha$ radiation source $(\lambda \approx 1.54 \AA)$ in the range of $0.5-5.5^{\circ}$. The elemental analysis was performed on an X-ray photoelectron spectroscopy instrument (XPS AXIS Ultra Kratos Analytical Ltd). The BET specific surface area $\left(S_{\mathrm{BET}}\right)$ and Barrett-JoynerHalenda $(\mathrm{BJH})$ pore size distribution were measured on a NOVA4200e surface area analyzer by using the $\mathrm{N}_{2}$ gas adsorption/desorption isotherm. Fourier transform infrared (FTIR) spectra were recorded by a FTIR-8400S spectrometer. The samples and $\mathrm{KBr}$ were pressed to form thin plates (the mass of $\mathrm{KBr}$ and the as-prepared sample was about $200 \mathrm{mg}$ and $2 \mathrm{mg}$, respectively) for obtaining the FTIR spectra. Thermogravimetric analysis (TGA) was carried out using a F2009 TG analyzer in the range of $25-900{ }^{\circ} \mathrm{C}$ at a heating rate of $10{ }^{\circ} \mathrm{C} \mathrm{min}{ }^{-1}$ under $\mathrm{N}_{2}$ flow. The zeta potential of the samples was measured by using a Zetasizer ZEN 3500 potentiometer in the $\mathrm{pH}$ range of 7-13.

\subsection{Electrochemical measurements}

The cyclic voltammetry (CV) curves and the electrochemical impedance spectra (EIS) of the MCMG samples were measured by a CHI660C electrochemical workstation using the twoelectrode method. The working electrodes were fabricated by mixing the prepared powders with acetylene black and polyvinylidene fluoride (PVDF) binder and the mass ratio was $8: 1: 1$. The appropriate amount of $N$-methylpyrrolidone (NMP) was added into the mixture to form a paste. Subsequently, the mixture was pressed onto a nickel foam and dried at $100{ }^{\circ} \mathrm{C}$ for $12 \mathrm{~h}$ in a vacuum oven. The active area of the electrode was about $1 \mathrm{~cm}^{2}$, the mass loading of the active materials was about $5 \mathrm{mg}$, and $4 \mathrm{M} \mathrm{KOH}$ solution was used as the electrolyte. According to the symmetric supercapacitor, two carbon-based electrodes with substantially equal mass were assembled into a button cell. The potential for CV was in the range of $0-0.8 \mathrm{~V}$ at the scanning rate of $10-200 \mathrm{mV} \mathrm{s}^{-1}$. The EIS was tested in the frequency range of $10^{-2}-10^{5} \mathrm{~Hz}$. The galvanostatic chargedischarge (GCD) and cycle measurements were performed on the LANDdt V7 test system. The current density was in the range of $0.1-2 \mathrm{~A} \mathrm{~g}^{-1}$ and the potential was in the range of $0-1 \mathrm{~V}$ during the GCD measurements.

\section{Results and discussion}

\subsection{Morphologies of the as-prepared composites}

The SEM images of the MCMG precursors obtained at different aging temperatures are shown in Fig. 1. Obviously, there are a large number of carbon particles with the diameter of about 30-40 nm on the surface of the MCMG precursors, which are cross-linked with each other similar to lamella depending on the GO surface and assembled to form interconnected macropores in the MCMG precursors. ${ }^{19,27}$ Furthermore, the size of the particles in the MCMG-140-0.2 precursor is smaller than that in the MCMG-130-0.2 precursor. Therefore, the morphologies of the MCMG precursors illustrate that the introduction of GO plays a guiding role in the formation of the lamella.

The SEM images of the as-prepared MCMG obtained with different CTAB concentrations at the aging temperature of $140{ }^{\circ} \mathrm{C}$ are shown in Fig. 2. It can be observed that the MCMG samples incorporated the layered and spherical materials. The wrinkled layered portion in the samples represents the reduced graphene formed via the high-temperature carbonization (as shown in Fig. 2(a-c)). Among these samples, the quasi-spherical materials on the surface of the MCMG is because the mesoporous carbon spheres are not formed when the concentration of CTAB was low (the concentration was $3.1 \mathrm{~g} \mathrm{~L}^{-1}, 5.3 \mathrm{~g} \mathrm{~L}^{-1}$, and $7.9 \mathrm{~g} \mathrm{~L}^{-1}$ ). Nevertheless, the spherical materials on the surface of the MCMG samples were significantly observed when the concentration of CTAB was high (as shown in Fig. 2(d-f)). When the concentration of CTAB was $10.6 \mathrm{~g} \mathrm{~L}^{-1}$, the spherical particles with uniform distribution have the diameter of about 30-40 $\mathrm{nm}$. There are inhomogeneous particles with the diameter of about $20-300 \mathrm{~nm}$ in the sample of MCMG-140-0.24 (the CTAB concentration was $12.6 \mathrm{~g}$ $\mathrm{L}^{-1}$ ). When the mass of the added CTAB increased to $0.3 \mathrm{~g}$ (the concentration was $15.8 \mathrm{~g} \mathrm{~L}^{-1}$ ), the surface of the obtained MCMG-140-0.3 was covered with uneven spherical particles with a large diameter of $200-800 \mathrm{~nm}$. The increase in the diameter of the carbon spheres is due to strong electrostatic interactions between $\mathrm{CTAB}$ and AMP with a higher concentration of $\mathrm{CTAB}$, which is similar to the previous report. ${ }^{39}$

The TEM images of the as-prepared MCMG samples are shown in Fig. 3. The surface of MCMG-130-0.2 has lamellar structures and irregular mesopores (as shown in Fig. 3(a and f)), which is due to the collapse of the precursor structure via carbonization at $900{ }^{\circ} \mathrm{C}$. In addition, these irregular and

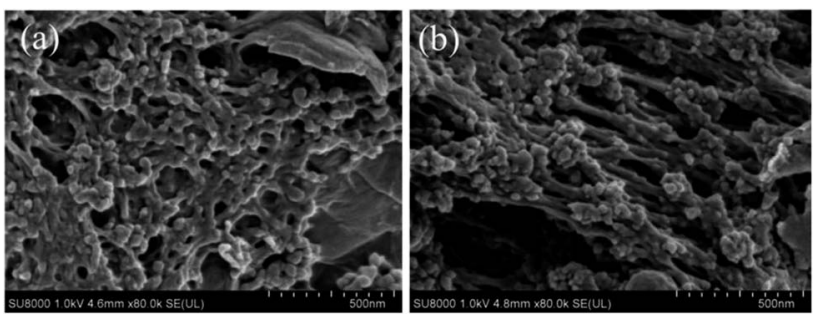

Fig. 1 SEM images of (a) the MCMG-130-0.2 precursor and (b) the MCMG-140-0.2 precursor. 

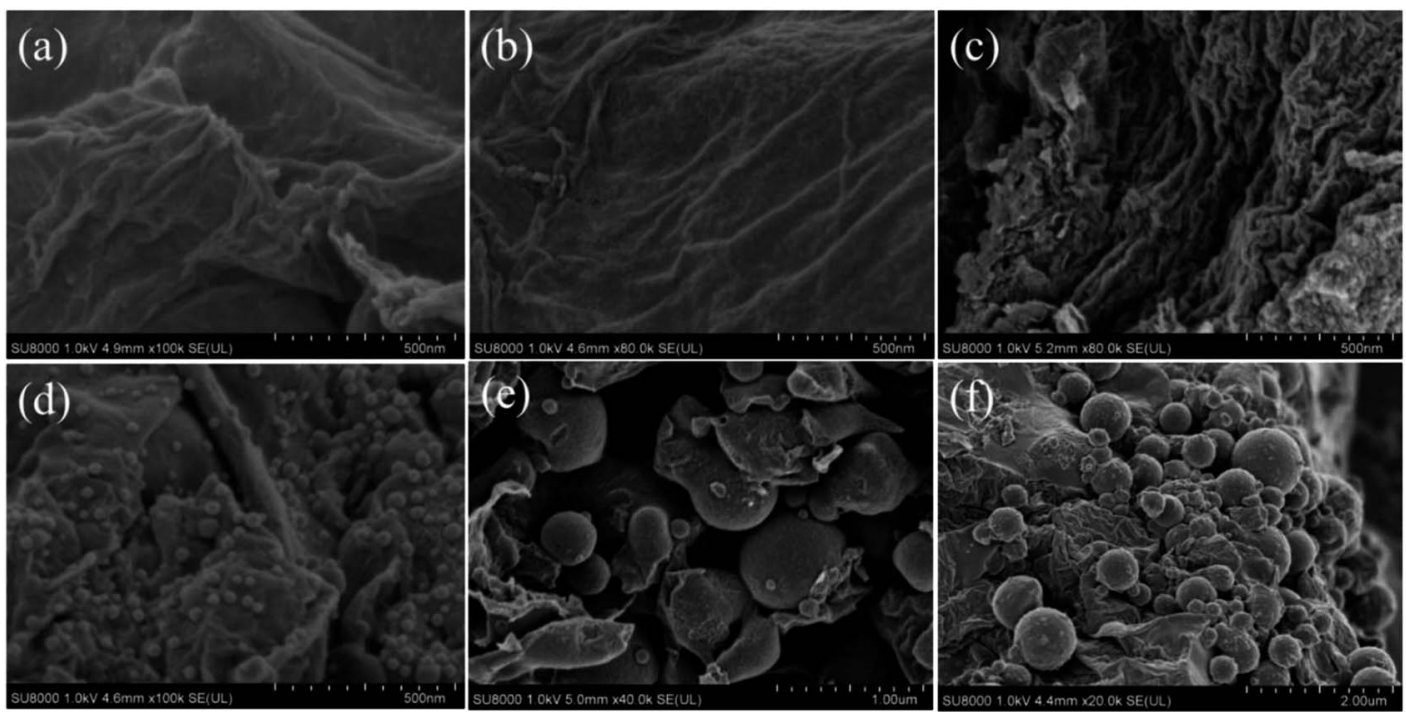

Fig. 2 SEM images of (a) MCMG-140-0.06, (b) MCMG-140-0.1, (c) MCMG-140-0.15, (d) MCMG-140-0.2, (e) MCMG-140-0.24, and (f) MCMG$140-0.3$.

disordered mesopores were derived from the precursor formed by CTAB and AMP. The MCMG-140-0.2 has carbon spheres among the graphenes (as shown in Fig. 3(c)), which constructed the sandwich composites with layered graphenes. Furthermore, the microsphere consists of many worm-like and partially ordered mesopores (as shown in Fig. $3(\mathrm{~g})$ ). According to the high-resolution images of MCMG-130-0.2 and MCMG-140-0.2, it is further proved that the MCMG samples obtained at the aging temperature of $130{ }^{\circ} \mathrm{C}$ and $140{ }^{\circ} \mathrm{C}$ have many mesopores. The MCMG-140-0.2 synthesized via self-assembly at the aging temperature of $140{ }^{\circ} \mathrm{C}$ shows a sandwich structure constructed by the mesoporous carbon spheres and graphenes. However, there are no obvious microspheres in the sample obtained at the aging temperature of $130^{\circ} \mathrm{C}$. The particles in MCMG-140-0.1 and MCMG-140-0.15 are more agglomerated and the graphenes are stacked unevenly (as shown in Fig. 3(b and c)). When the concentration of CTAB was in the range of 10.6-12.6 $\mathrm{g} \mathrm{L}^{-1}$, the carbon spheres with different diameters (as shown in Fig. 3(d and e)) are uniformly distributed among the layered graphenes, indicating that MCMG with sandwich structure have been successfully synthesized. However, the sample of MCMG-1400.24 contains a few carbon microspheres with the diameter of several hundred nanometers. The TEM results are in accordance with the SEM analysis.
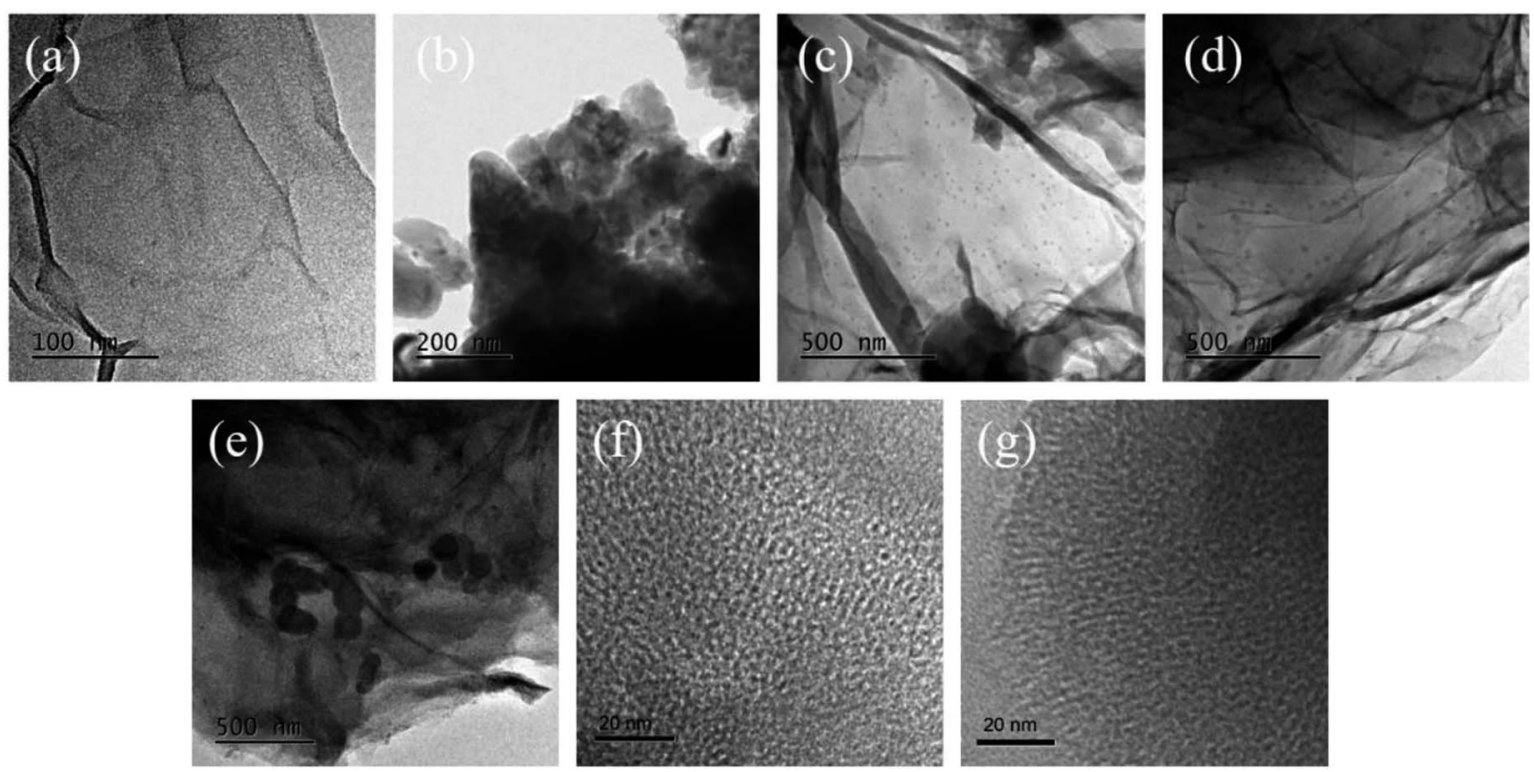

Fig. 3 TEM images of (a) MCMG-130-0.2, (b) MCMG-140-0.1, (c) MCMG-140-0.15, (d) MCMG-140-0.2, (e) MCMG-140-0.24, the high resolution TEM images of (f) MCMG-130-0.2 and (g) MCMG-140-0.2. 

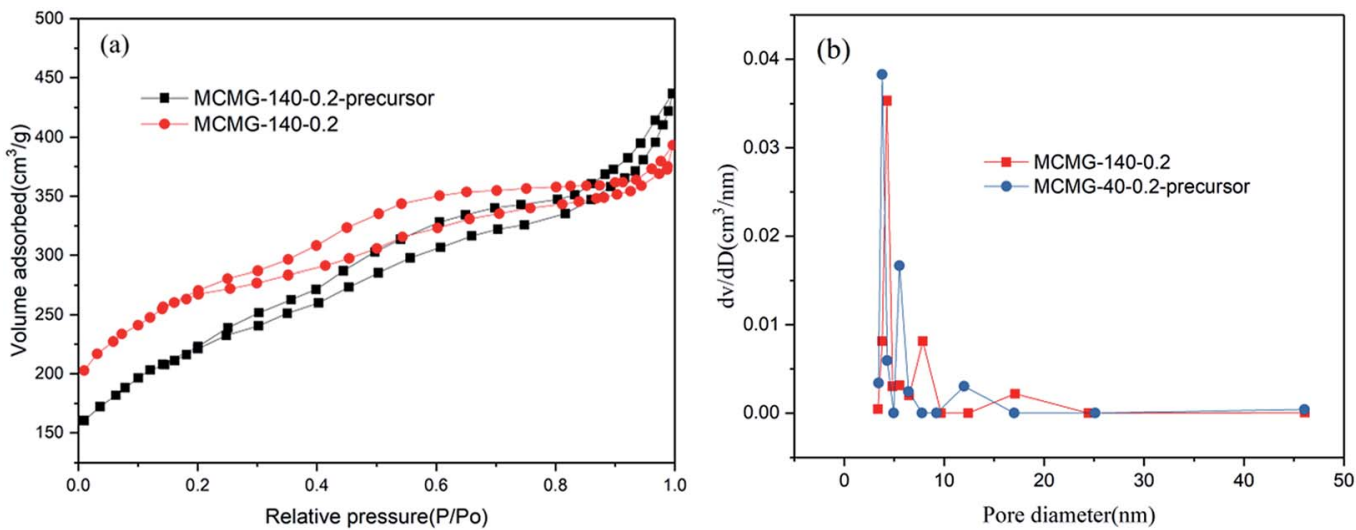

Fig. 4 (a) $\mathrm{N}_{2}$ adsorption-desorption isotherms and (b) the pore size distribution of the MCMG-140-0.2 precursor and MCMG-140-0.2.

\subsection{Pore structure and microstructure of the MCMG precursor and MCMG}

The nitrogen adsorption-desorption isotherms and pore size distribution curves of the MCMG-140-0.2 precursor and MCMG140-0.2 are shown in Fig. 4. Both of the isotherms exhibit type IV adsorption curve and $\mathrm{H}_{2}$ type hysteresis loop (as shown in Fig. 4(a)), indicating that the two samples have a large amount of mesopores with a slit shape. ${ }^{35,39}$ This is consistent with the TEM result for MCMG-140-0.2 in Fig. 3(g). In the relative pressure region of $0.3-0.9$, the MCMG-140-0.2 precursor shows a bigger loop, indicating that more mesopores exist in this MCMG sample. Moreover, some macropores exist in the MCMG precursor and the MCMG sample, according to the curve in the high pressure region $\left(P / P_{0}>0.9\right)$. The $S_{\mathrm{BET}}$ and pore volume for the MCMG-140-0.2 precursor and MCMG-140-0.2 are $463.4 \mathrm{~m}^{2}$ $\mathrm{g}^{-1}, 0.51 \mathrm{~cm}^{3} \mathrm{~g}^{-1}$ and $1150.5 \mathrm{~m}^{2} \mathrm{~g}^{-1}, 0.86 \mathrm{~cm}^{3} \mathrm{~g}^{-1}$, respectively. The higher $S_{\mathrm{BET}}$ and larger pore volume of MCMG-140-0.2 suggest the formation of more mesopores and micropores in the MCMG sample during the carbonization of the MCMG precursor. According to the $\mathrm{BJH}$ pore size distribution curve (as shown in Fig. 4(b)), the pore size of the MCMG-140-0.2 precursor is centered at $3.8 \mathrm{~nm}, 5.5 \mathrm{~nm}$, and $11.9 \mathrm{~nm}$. In addition, the pore size of MCMG-140-0.2 is centered at $4.3 \mathrm{~nm}$, $7.9 \mathrm{~nm}$, and $17.1 \mathrm{~nm}$. Compared with the results, the carbon skeleton shrinks during carbonizing but the pore size increases a little due to the elimination of light components such as $\mathrm{CO}_{2}$, $\mathrm{NH}_{3}, \mathrm{NO}_{2}$, small molecular alkenes and alkanes. However, it is very difficult to observe large mesopores (such as $17.1 \mathrm{~nm}$ ), according to the TEM images for the MCMG samples.

The SAXRD patterns of the as-prepared samples are shown in Fig. 5. The samples of the MCMG precursors show an obvious diffraction peak at around $2^{\circ}$ (as shown in Fig. 5(a)) that can be indexed as the (210) reflection of the cubic structure, suggesting that they contain long-range ordered lyotropic liquid crystals. ${ }^{29,40}$ In addition, these samples show a weak diffraction peak at around $3.9^{\circ}$, indicating that the short-range ordered mesopores may exist in the MCMG precursors. The intensity of the diffraction peak increases with an increase in the CTAB content, indicating that the ordered degree of the mesopores is improved due to the sufficient self-assembly between the AMP and CTAB molecules. Nevertheless, the diffraction peaks of the MCMG samples shift to a higher angle (about 2.3 ) and the intensity is weakened (as shown in Fig. 5(b)), which is due to the shrinkage of the carbon skeleton and the collapse of the partial pore channels during carbonization at $900{ }^{\circ} \mathrm{C}$. Therefore, the
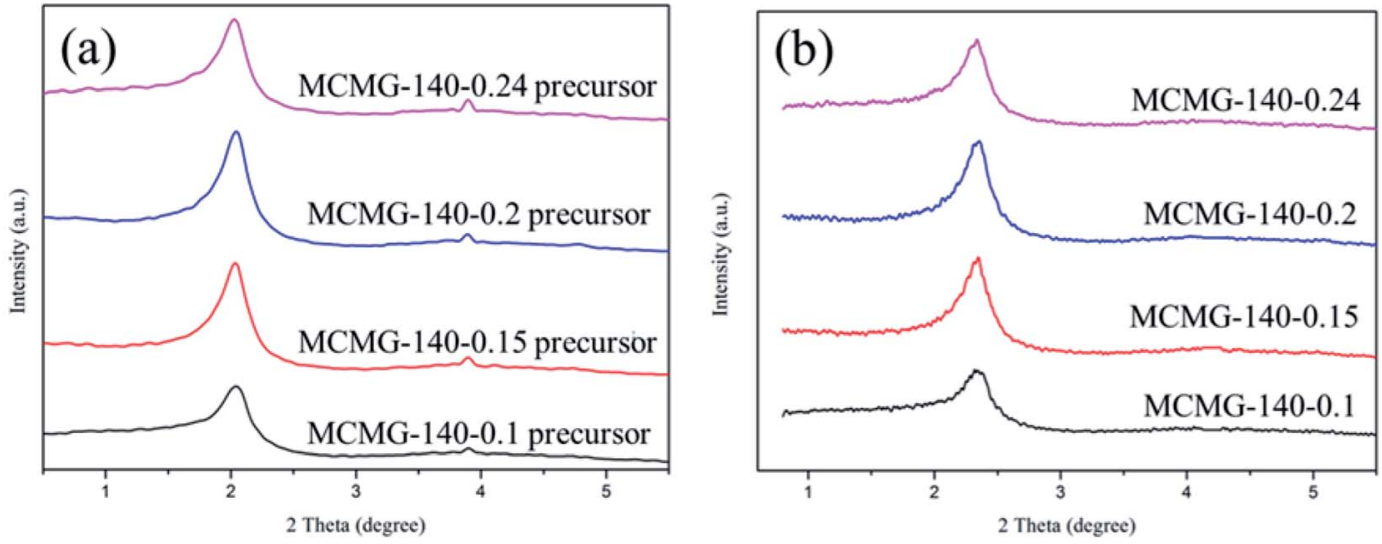

Fig. 5 SAXRD spectra of (a) the MCMG precursors and (b) the MCMG samples obtained at $900{ }^{\circ} \mathrm{C}$. 

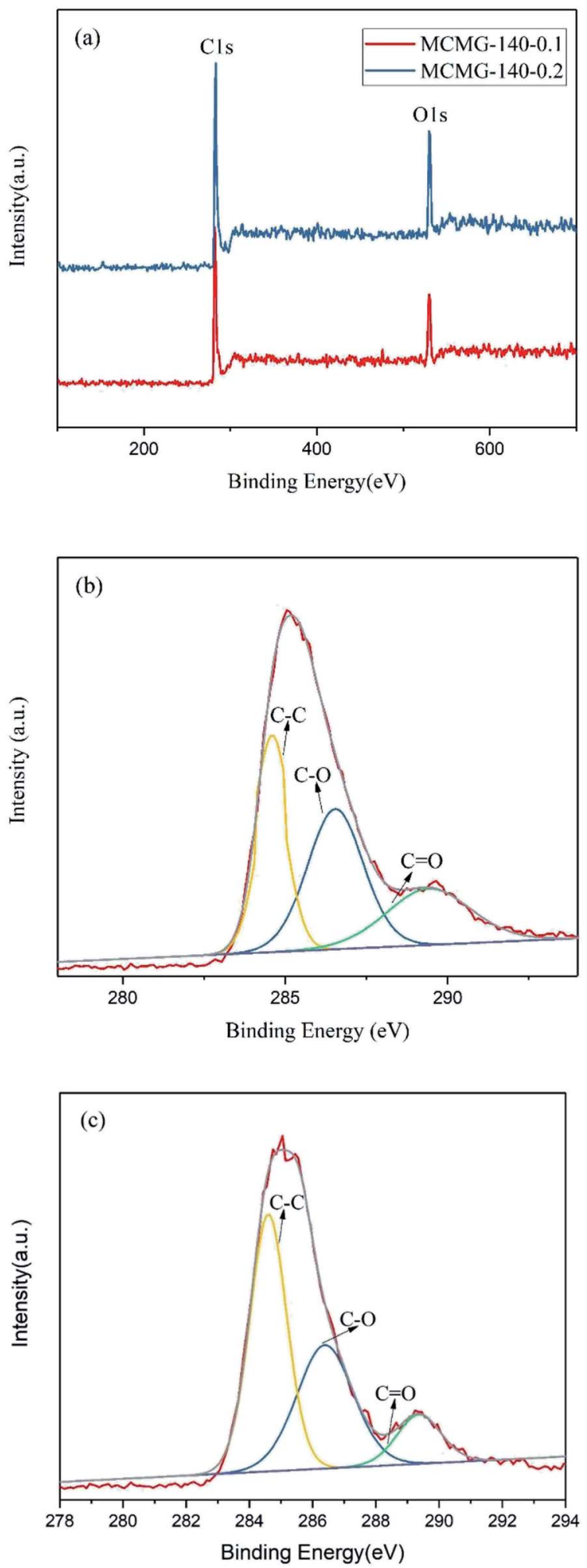

Fig. 6 (a) XPS spectra of MCMG-140-0.1 and MCMG-140-0.2, (b) C 1s spectra of MCMG-140-0.1, (c) C 1s spectra of MCMG-140-0.2.

ordered degree of the mesopores in the MCMG reduces. When the mass ratios of AMP to CTAB were $3: 2$ and $5: 4$, the diffraction peak for the synthesized MCMG samples at $2.3^{\circ}$ is sharp. Therefore, the CTAB concentration in the range of 10.6-

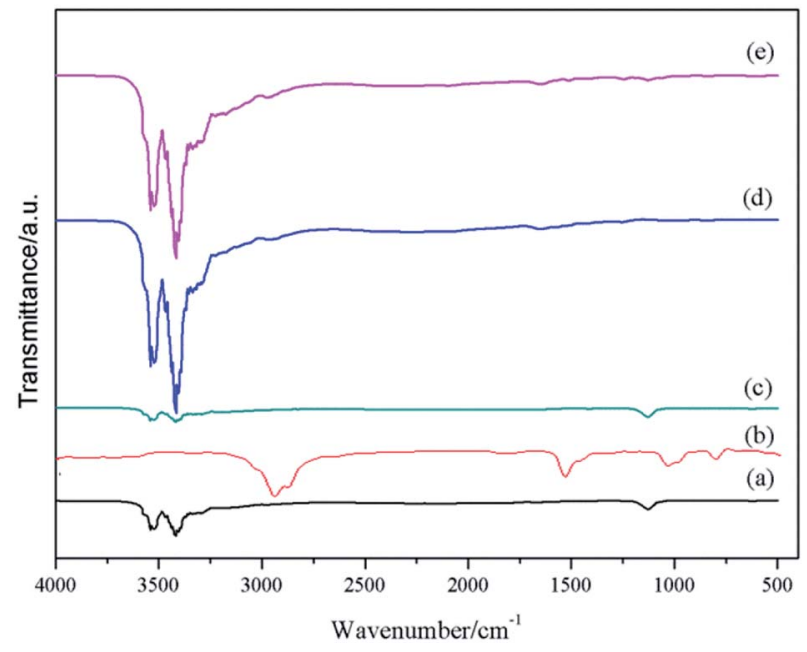

Fig. 7 FTIR spectra of (a) AMP, (b) CTAB, (c) MCMG-140-0.2, (d) GO, and (e) MCMG-140-0.2 precursor.

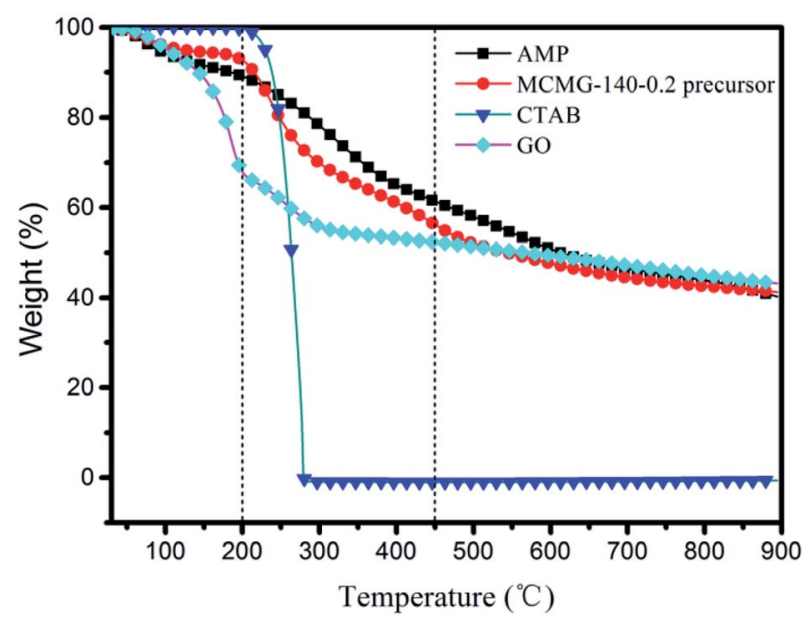

Fig. 8 TGA curves of AMP, CTAB, GO, and MCMG-140-0.2 precursor.

$12.6 \mathrm{~g} \mathrm{~L}^{-1}$ is favorable for forming the mesoporous structure in MCMG.

The XPS spectra of MCMG-140-0.1 and MCMG-140-0.2 are shown in Fig. 6. The full XPS spectra for MCMG-140-0.1 and MCMG-140-0.2 are almost the same (as shown in Fig. 6(a)), and the characteristic peaks at around 283 and $530 \mathrm{eV}$ represent $\mathrm{C} 1 \mathrm{~s}$ and $\mathrm{O} 1 \mathrm{~s}$, respectively. Furthermore, three fitting peaks for $\mathrm{C} 1 \mathrm{~s}$ at $284.6,286.3$, and $289.3 \mathrm{eV}$ in the fitting curves (as shown in Fig. 6(b and c) ) correspond to $\mathrm{C}-\mathrm{C}, \mathrm{C}-\mathrm{O}$, and $\mathrm{C}=\mathrm{O}$, respectively. ${ }^{41}$ In addition, the oxygen content for MCMG-140-0.1 and MCMG-140-0.2 is 5.4 at\% and 4.9 at\%, respectively. Therefore, the compositions of the as-prepared MCMG samples have no obvious differences except for the different contents of oxygen.

The FTIR spectra of the raw materials, MCMG-140-0.2 precursor, and MCMG-140-0.2 are shown in Fig. 7. According to the spectrum of the MCMG-140-0.2 precursor, the absorption bands at $2850-3000 \mathrm{~cm}^{-1}$ are ascribed to the stretching vibration of methyl and methylene, which are the characteristic 

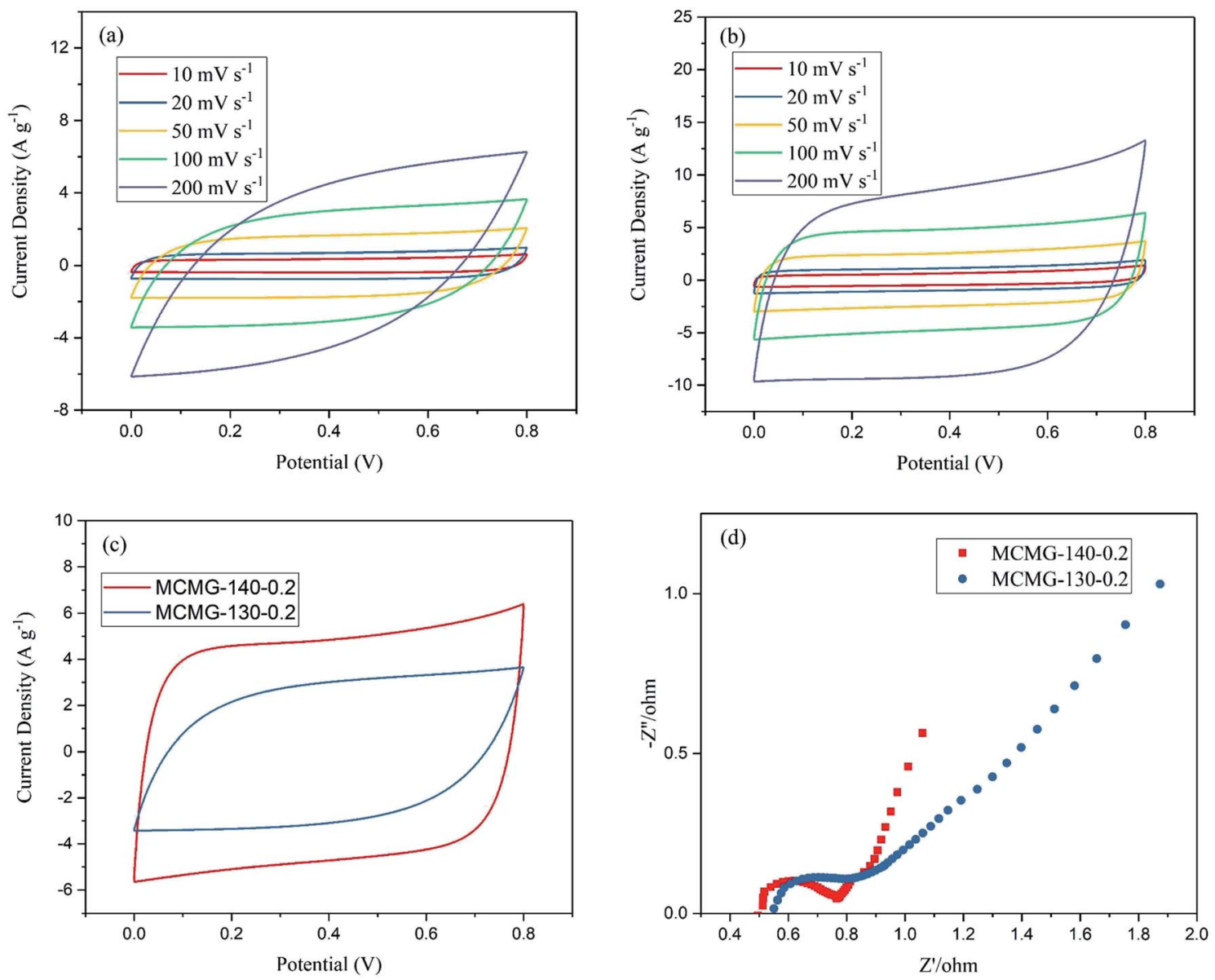

Fig. 9 (a) CV curves of MCMG-130-0.2 at different scanning rates, (b) CV curves of MCMG-140-0.2 at different scanning rates, (c) CV curves of MCMG-130-0.2 and MCMG-140-0.2 at the scanning rate of $100 \mathrm{mV} \mathrm{s}^{-1}$, (d) EIS plots of MCMG-130-0.2 and MCMG-140-0.2.

absorption peaks of CTAB. ${ }^{42}$ In addition, the absorption peak at about $1123 \mathrm{~cm}^{-1}$ is ascribed to the stretching vibration of carbonyl in AMP and GO. ${ }^{39,43}$ The absorption bands at 3000$3522 \mathrm{~cm}^{-1}$ in the spectra of GO and MCMG-140-0.2 are ascribed to the stretching vibration of hydroxyl and hydrogen bonds. The FTIR spectrum of AMP is similar to that of the MCMG-140-0.2 precursor. Compared with the MCMG-140-0.2 precursor, the absorption peak of MCMG-140-0.2 at $2900 \mathrm{~cm}^{-1}$ disappears but the weak absorption peaks in the range of $3000-3500 \mathrm{~cm}^{-1}$ remain, indicating that the structure-directing agent СТАВ has been removed via the carbonization process.

\subsection{Thermal analysis of the samples}

As shown in Fig. 8, TGA of GO, CTAB, AMP, and MCMG-140-0.2 precursors was carried out in the temperature range of 25$900{ }^{\circ} \mathrm{C}$ at a heating rate of $10{ }^{\circ} \mathrm{C} \mathrm{min}^{-1}$ under $\mathrm{N}_{2}$ flow. CTAB exhibits $100 \mathrm{wt} \%$ weight loss in the range of $200-285{ }^{\circ} \mathrm{C}$, indicating that the structure-directing agent is decomposed completely before $300{ }^{\circ} \mathrm{C}$. In the range of $25-230{ }^{\circ} \mathrm{C}$, the weight loss of AMP, GO, and the MCMG-140-0.2 precursor is mainly ascribed to the removal of adsorbed $\mathrm{H}_{2} \mathrm{O}$. Compared with AMP, the weight loss of the MCMG-140-0.2 precursor is lower before $230{ }^{\circ} \mathrm{C}$, which may be ascribed to the restriction of porous structure to $\mathrm{H}_{2} \mathrm{O}$. In the range of $230-500{ }^{\circ} \mathrm{C}$, some small molecules are removed and partial oxygen-containing functional groups begin to decompose in AMP (29.8 wt\% weight loss).$^{39}$ Importantly, in the range of $230-285^{\circ} \mathrm{C}$, the weight loss of MCMG-140-0.2 is higher than that of AMP due to the decomposition of CTAB. Compared with GO, the weight loss of the MCMG-140-0.2 precursor is lower before $500{ }^{\circ} \mathrm{C}$, which may be due to the strong electrostatic interactions between the AMP molecules and the GO/CTAB composites. When the temperature exceeded $600{ }^{\circ} \mathrm{C}$, GO, AMP, and the MCMG-140-0.2 precursor underwent slow weight loss, mainly corresponding to the removal of heteroatoms. ${ }^{44}$ The results of TGA show that CTAB can be effectively removed by carbonization at $900^{\circ} \mathrm{C}$ and the open pores in MCMG are thus obtained. Furthermore, the TGA results are in accordance with the FTIR analysis.

\subsection{Electrochemical performances of the MCMG samples}

Fig. 9 shows the CV curves and EIS plots of MCMG-130-0.2 and MCMG-140-0.2 using $4 \mathrm{M} \mathrm{KOH}$ solution as the electrolyte. We find that all of the CV curves for the two samples remain quasirectangular in shape in the potential range of $0-0.8 \mathrm{~V}$ at the scanning rate of $10-200 \mathrm{mV} \mathrm{s}^{-1}$ and the electrolyte shows no dissociation, according to the CV curves (as shown in Fig. 9(a 

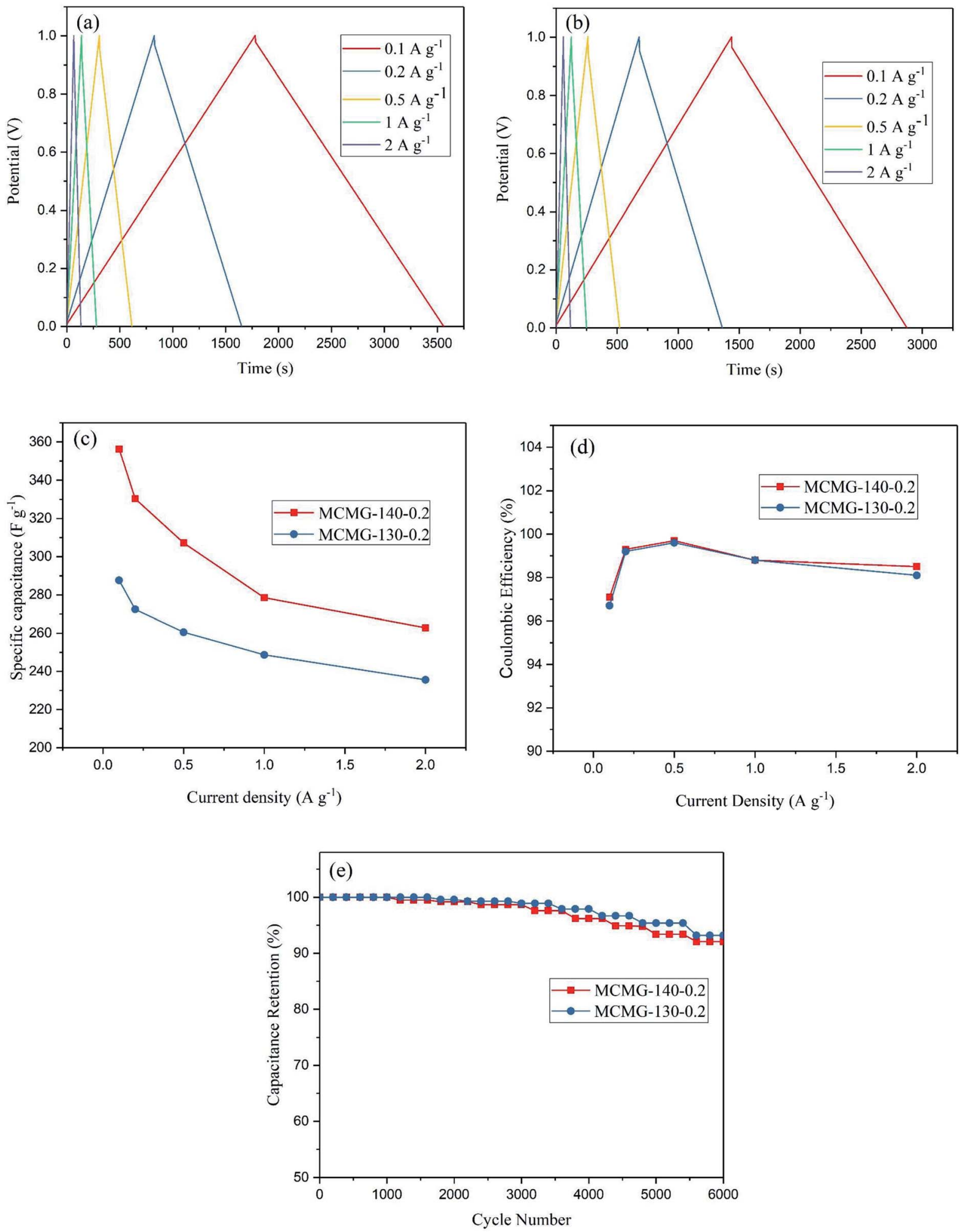

Fig. 10 (a) GCD curves of MCMG-140-0.2 at different current densities, (b) GCD curves of MCMG-130-0.2 at different current densities, (c) the specific capacitance of MCMG-130-0.2 and MCMG-140-0.2 at different current densities, (d) the coulombic efficiency of MCMG-130-0.2 and MCMG-140-0.2 at different current densities, (e) the capacitance retention of MCMG-130-0.2 and MCMG-140-0.2 during 6000 chargedischarge cycles.

and b)), suggesting an excellent capacitive behavior and a high stability for the electrodes. To distinguish the electrochemical performance of MCMG-140-0.2 and MCMG-130-0.2, the CV curves of the two samples at $100 \mathrm{mV} \mathrm{s}^{-1}$ are shown in Fig. 9(c). We found that the CV curve area for MCMG-140-0.2 is larger than that for MCMG-130-0.2, confirming that the former 
Table 1 Comparison of the specific capacitance in this work with other data in the published literature

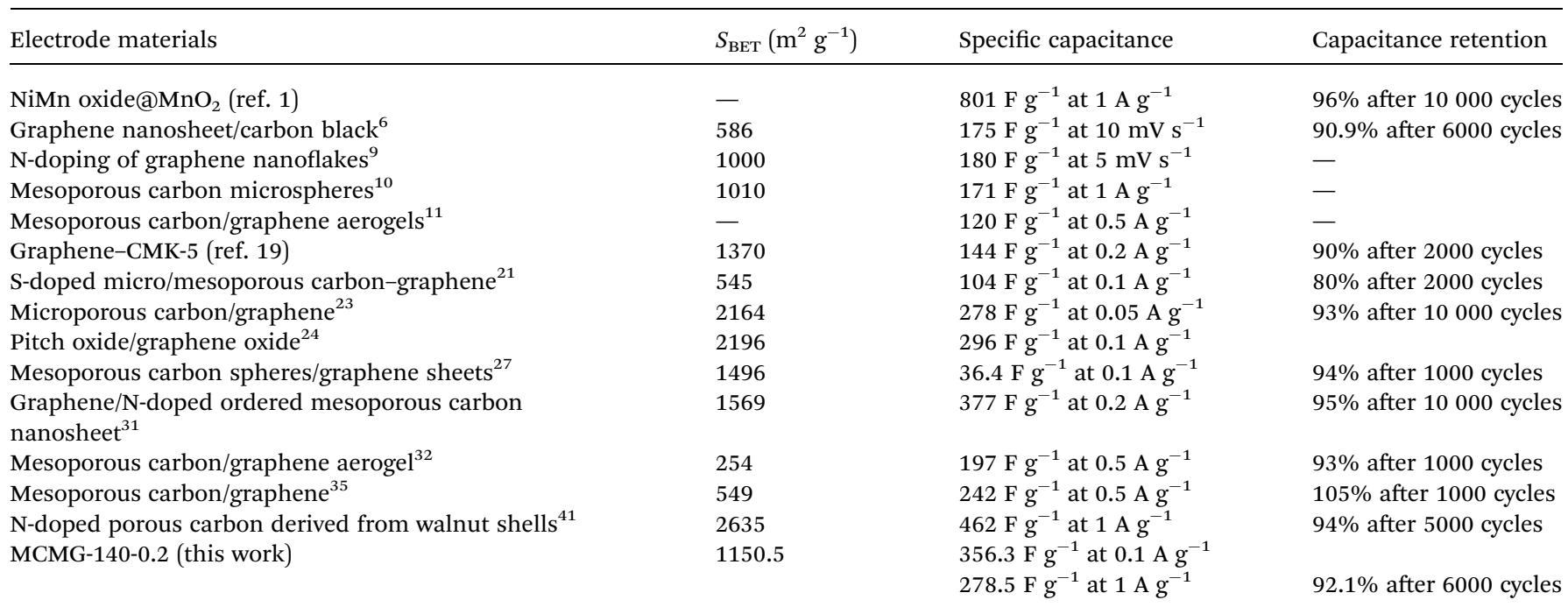

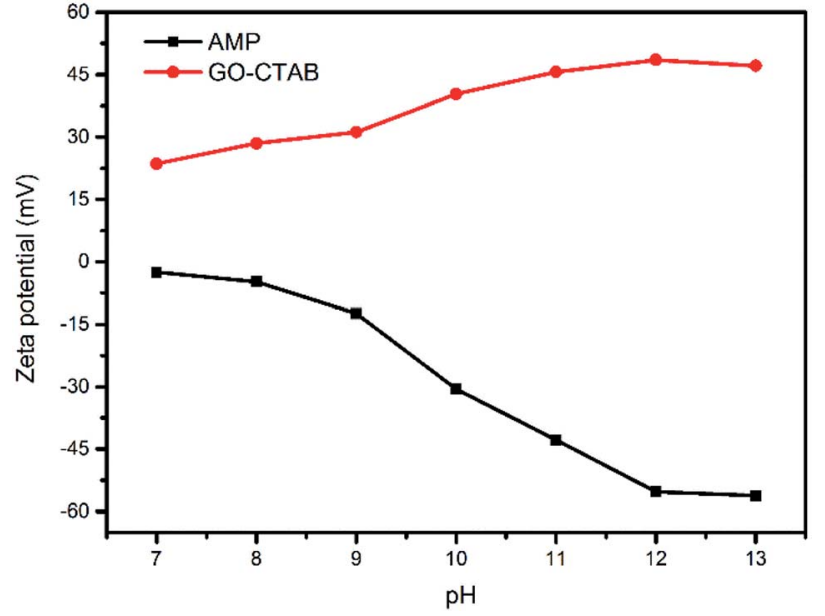

Fig. 11 Zeta potential of AMP and GO/CTAB dispersions in the $\mathrm{pH}$ range of $7-13$.

possesses a higher specific capacitance. According to the results of TEM and nitrogen adsorption-desorption isotherms, the formed mesoporous carbon microspheres separate the graphene layers well for the MCMG-140-0.2 sample, providing a high specific surface area and abundant mesopores, which is beneficial for the improvement of specific capacitance for the sample.

The EIS plots of MCMG-130-0.2 and MCMG-140-0.2 are shown in Fig. 9(d). It is noted that the high slope and the line morphology imply that the as-prepared samples with many pores are good for use as electrode materials in supercapacitors. ${ }^{45}$ We found that the slopes for MCMG-130-0.2 and MCMG-140-0.2 in the low frequency region show obvious differences, and the line for the latter is closer to the imaginary axis, indicating that the MCMG-140-0.2 sample will have a better electrochemical performance. The equivalent series resistance $\left(R_{\mathrm{S}}\right)$ is the sum of the resistance of the electrolyte and the intrinsic resistance of the active materials, and the interfacial contact resistance between the active materials and the current collector ${ }^{46}$ the intersection on the real axis in the high frequency region represents the value of $R_{\mathrm{S}}$. In addition, the diameter of the semicircle in the high frequency region represents the value of interface resistance $\left(R_{\mathrm{ct}}\right)$. The $R_{\mathrm{s}}$ of MCMG140-0.2 and MCMG-130-0.2 is $0.51 \Omega$ and $0.55 \Omega$, respectively, and we find that the former is lower than that for many carbon materials used in the supercapacitors. Moreover, the $R_{\mathrm{ct}}$ of MCMG-140-0.2 and MCMG-130-0.2 is about $0.26 \Omega$ and $0.29 \Omega$, respectively, both of which are small.

The GCD curves for the two samples at the current density of 0.1-2 $\mathrm{A} \mathrm{g}^{-1}$ are shown in Fig. 10(a and b). These curves show symmetric consistency at different current densities, indicating that as-prepared MCMG samples have excellent stability and reversibility of electrochemical performance for supercapacitors. The specific capacitances at the current densities were calculated according to eqn (1) in our previous reports: $:^{47,48}$

$$
C_{\mathrm{m}}=\frac{2 I \Delta t}{m \Delta V}
$$

where $I$ is the current loaded (A), $\Delta t$ is the discharge time (s), $m$ is the mass of active substances $(\mathrm{g})$, and $\Delta V$ is the potential window (V). The calculated results are shown in Fig. 10(c). We find that the specific capacitance of MCMG-140-0.2 was as high as $356.3 \mathrm{~F} \mathrm{~g}^{-1}$ at the current density of $0.1 \mathrm{Ag}^{-1}$ and it remained at $262.8 \mathrm{~F} \mathrm{~g}^{-1}$ at the current density of $2 \mathrm{~A} \mathrm{~g}^{-1}$. In addition, the specific capacitance of MCMG-140-0.2 was $287.6 \mathrm{~F} \mathrm{~g}^{-1}$ at $0.1 \mathrm{~A} \mathrm{~g}^{-1}$ and it remained at $235.6 \mathrm{~F} \mathrm{~g}^{-1}$ at $2 \mathrm{~A} \mathrm{~g}^{-1}$. The specific capacitance of MCMG-140-0.2 is higher than that of MCMG-1300.2 , which is consistent with the results of CV curve analysis. In addition, the coulombic efficiency for MCMG-140-0.2 and MCMG-130-0.2 first increased and then decreased with the increase in the current density (as shown in Fig. 10(d)); they reached $99.7 \%$ and $99.6 \%$ at $0.5 \mathrm{~A} \mathrm{~g}^{-1}$, and both of them 


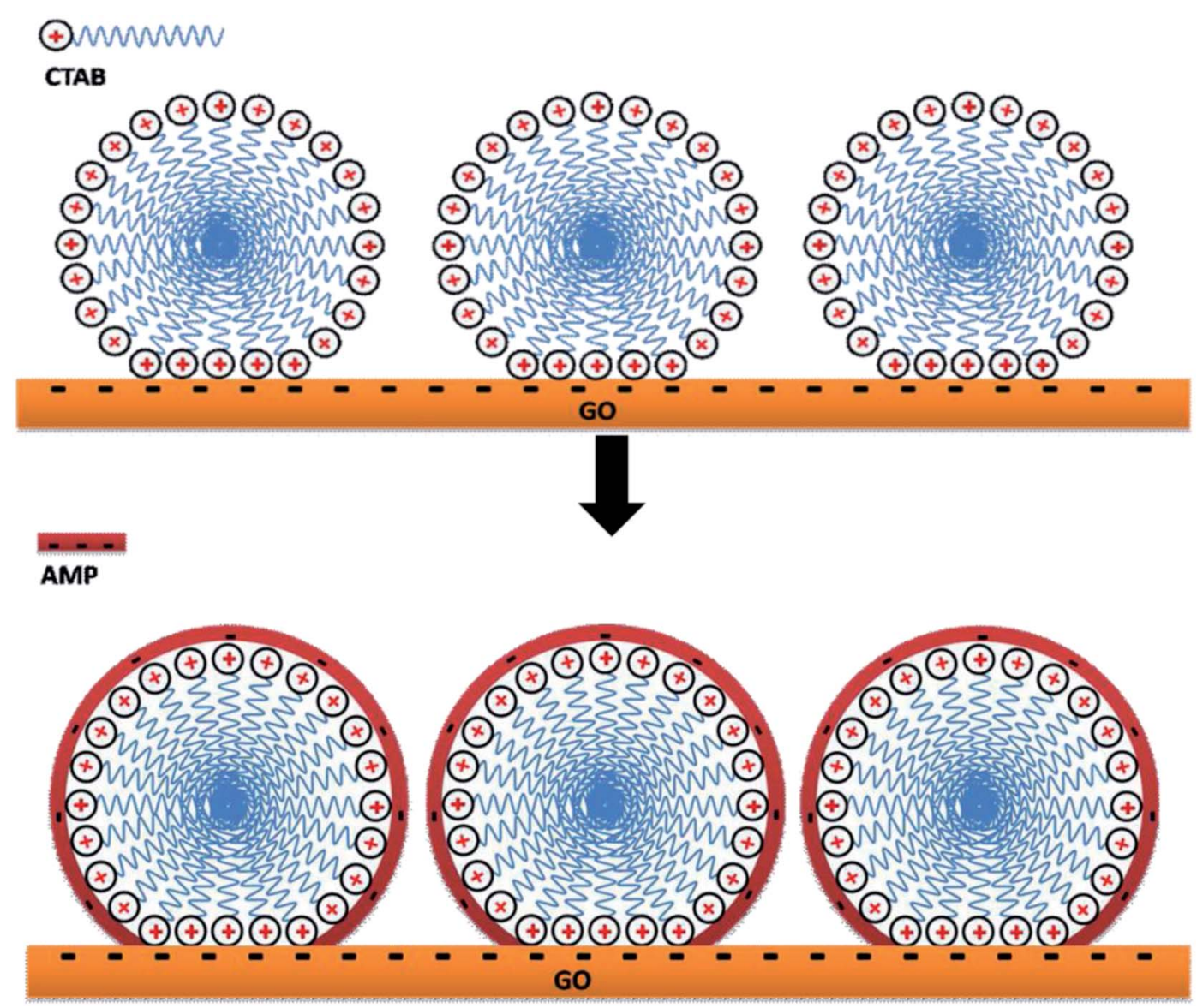

Fig. 12 Schematic diagram of the interaction between AMP, CTAB, and GO.

remained over $98 \%$ at $2 \mathrm{~A} \mathrm{~g}^{-1}$. Therefore, the coulombic efficiency of MCMG is still high at a high current density.

To investigate the cycling stability of the as-prepared MCMG samples, the charge-discharge cycling of MCMG-140-0.2 and MCMG-130-0.2 were tested at the current density of $1 \mathrm{~A} \mathrm{~g}^{-1}$, and the capacitance retention of the two samples during the cycling is shown in Fig. 10(e). The capacitance retention of MCMG-1400.2 and MCMG-130-0.2 after 6000 cycles was $92.1 \%$ and $93.2 \%$, respectively. The comparison of the specific capacitance in this work with other materials in previous reports is shown in Table 1. We found that the specific capacitance for MCMG is higher than that for most carbon based electrode materials in previous studies and the retention is comparable with that for the mesoporous carbon/graphene composites. Moreover, the high retention after charge-discharge cycling and the high specific capacitance at a high current density are ascribed to the synergy between the graphenes and the mesoporous carbon spheres, indicating that the MCMG-140-0.2 sample possesses excellent cycling stability and rate capability.

It is noted that the ordered mesopores in electrode materials can not only increase their specific surface area but are also beneficial for providing channels for the rapid transport of ions in supercapacitors. ${ }^{\mathbf{4 9 , 5 0}}$ Moreover, small mesopores (2-4 nm) have long diffusion paths for ions, which has negative effects on the electrochemical performances of the electrode materials.
Therefore, ordered mesorpores with appropriate pore size in electrode materials are desired for supercapacitors. In this paper, the as-prepared MCMG samples have many mesopores; however, the mesopores in MCMG-130-0.2 are irregular and disordered and that in MCMG-140-0.2 are partially ordered, according to the TEM images. Furthermore, the pore sizes of MCMG-140-0.2 are appropriate and centered at $4.3 \mathrm{~nm}, 7.9 \mathrm{~nm}$, and $17.1 \mathrm{~nm}$, according to the nitrogen adsorption-desorption isotherm. According to the CV and GCD curves of MCMG-1400.2 , a quasi rectangle and symmetric isosceles triangle with an increase in the scan rate and current density is maintained, indicating that the partially ordered mesopores in MCMG-1400.2 are beneficial for maintaining the double electrode layer characteristic. In addition, the $R_{\text {ct }}$ of MCMG-130-0.2 is higher than that for MCMG-140-0.2. This is because poor regularity and many defects in the mesopores in MCMG-130-0.2 increase the interface resistance. Therefore, partially ordered mesopores with appropriate pore size in the MCMG samples have important effects on improving their electrochemical performances.

\subsection{Formation mechanism of MCMG}

The GO dispersion was negatively charged over a wide $\mathrm{pH}$ range due to the ionization of carboxylic acid and hydroxyl groups on the GO surface. ${ }^{51}$ The $\mathrm{pH}$ is beyond 7 because AMP can only be 
dissolved in an alkaline solution. After the addition of CTAB, the GO/CTAB dispersion was inversed to be completely positively charged in the $\mathrm{pH}$ range studied in this work (as shown in Fig. 11) due to the adsorption of CTAB on the GO surface. The zeta potential of the $\mathrm{GO} / \mathrm{CTAB}$ dispersion changed with the variation in the $\mathrm{pH}$ value. The zeta potential of AMP was negatively charged, which is similar to that of the GO dispersion. ${ }^{27,51}$ The absolute value of the zeta potential of the samples first increases and then decreases with increasing $\mathrm{pH}$. The very high zeta potential of the positively charged $\mathrm{GO} / \mathrm{CTAB}$ is $+48.5 \mathrm{mV}$ at $\mathrm{pH}=12$ when the concentration of the GO solution was $0.6 \mathrm{mg}$ $\mathrm{mL}^{-1}$ and the concentration of CTAB was $10.6 \mathrm{~g} \mathrm{~L}^{-1}$ (the CTAB content was $0.2 \mathrm{~g}$ ). In addition, the very low zeta potential of the AMP dispersion was $-55.3 \mathrm{mV}$ at $\mathrm{pH}=12$ (the AMP content was $0.3 \mathrm{~g}$ ). Therefore, a strong electrostatic interaction between the $\mathrm{GO} / \mathrm{CTAB}$ composites and the AMP molecules at $\mathrm{pH}=12$ (in accordance with the conditions of the experiment in this work) can promote the latter to attach firmly on the surface of $\mathrm{GO}$ / CTAB by self-assembly. Then, the CTAB/AMP micelles with spherical shape are formed between the GO sheets, according to the surface energy minimum principle. The MCMG precursors are obtained via subsequent gelling and crosslinking process. The schematic diagram of the self-assembly among GO, CTAB, and AMP is shown in Fig. 12. Finally, the MCMG composites will be synthesized by carbonizing the MCMG precursors.

It is noted that the structure-directing agent can form a spherical micelle when its concentration is appropriate. ${ }^{39}$ When the concentration of CTAB is low, the size of the spherical micelle of CTAB/AMP is small, and the morphology of the obtained mesoporous carbon microspheres in MCMG is difficult to maintain and easily aggregates, according to the TEM images. The diameter of the mesoporous carbon microspheres increases and they can maintain the spherical shape with increasing the concentration of CTAB. When the concentration of CTAB is high, the diameter of the mesoporous carbon microspheres can reach several hundred nanometers. In addition, the concentration of CTAB may have an effect on the pore structure of the obtained mesoporous carbon microspheres. Therefore, the concentration of CTAB has an important role in the morphology of MCMG besides the zeta potential of $\mathrm{GO}$ / CTAB.

\section{Conclusions}

In this work, CTAB was used as the structure-directing agent, and AMP and GO were used as the carbon sources to prepare MCMG via a soft template method. At the aging temperature of $130{ }^{\circ} \mathrm{C}$, no spherical particles were obtained in the composites. Nevertheless, mesoporous carbon spheres obtained at $140{ }^{\circ} \mathrm{C}$ combine with the graphene layers to form MCMG with a sandwich structure after carbonization at $900{ }^{\circ} \mathrm{C}$. When the concentration of CTAB was less than $10.6 \mathrm{~g} \mathrm{~L}^{-1}$, the synthesized composites easily agglomerated and the carbon nanoparticles were dispersed disorderedly on the graphene surface. When the concentration of CTAB was in the range of 10.6-12.6 $\mathrm{g} \mathrm{L}^{-1}$, the mesoporous carbon spheres were uniformly dispersed among the layered graphenes. When the concentration of CTAB was very high, unevenly distributed mesoporous carbon spheres with a large diameter were observed in MCMG. Due to the introduction of graphene and the formation of mesoporous carbon spheres, the composites with many mesopores had a significantly large specific surface area. Furthermore, the asprepared MCMG composites possessed high specific capacitance, and exhibited excellent cycling stability and rate capability, indicating that the electrochemical performances of the composites can be improved by the synergy of graphenes and mesoporous carbon spheres with a small diameter.

\section{Conflicts of interest}

There are no conflicts to declare.

\section{Acknowledgements}

This work is supported by the National Natural Science Foundation of China (Grant No. 51772243) and the China Postdoctoral Science Foundation Funded Project (Grant No. 2016M592824).

\section{References}

1 X. L. Guo, T. Wang, T. X. Zheng, C. H. Xu, J. T. Zhang, Y. X. Zhang, X. Y. Liu and F. Dong, J. Mater. Chem. A, 2018, 6, 24717.

2 Z. S. Wu, W. C. Ren, D. W. Wang, F. Li, B. L. Liu and H. M. Cheng, ACS Nano, 2010, 4, 5835.

3 X. Guo, P. Liu, J. Han, Y. Ito, A. Hirata, T. Fujita and M. Chen, Adv. Mater., 2015, 27, 6137.

4 H. He and C. Gao, ACS Appl. Mater. Interfaces, 2010, 2, 3201.

5 Q. Liao, N. Li, S. Jin, G. Yang and C. Wang, ACS Nano, 2015, 9, 5310.

6 J. Yan, T. Wei, B. Shao, F. Q. Ma, Z. J. Fan, M. L. Zhang, C. Zheng, Y. C. Shang, W. Z. Qian and F. Wei, Carbon, 2010, 48, 1731.

7 U. N. Maiti, W. J. Lee, J. M. Lee, Y. Oh, J. Y. Kim, J. E. Kim, J. Shim, T. H. Han and S. O. Kim, Adv. Mater., 2014, 26, 40.

8 S. Grimme, M. A. Christian and J. Antony, J. Phys. Chem. C, 2007, 111, 11199.

9 E. A. Arkhipova, A. S. Ivanov, S. V. Savilov, K. I. Maslakov, S. A. Chernyak, Y. A. Tambovtseva and V. V. Lunin, Funct. Mater. Lett., 2018, 11, 184005.

10 W. Xiong, M. X. Liu, L. H. Gan, Y. K. Lv, Y. Li, L. Yang, Z. J. Xu, Z. X. Hao, H. L. Liu and L. W. Chen, J. Power Sources, 2011, 196, 10461.

11 N. Chandrasekaran, V. Premkumar, S. M. S. Kumar and R. Ram, New J. Chem., 2018, 42, 7371.

12 J. Qian, M. Zhou, Y. Cao, X. Ai and H. Yang, J. Phys. Chem. C, 2010, 114, 3477.

13 Z. Z. Li and Y. B. Tan, ChemistrySelect, 2018, 3, 4319.

14 R. P. Zheng and S. J. Liao, Surf. Technol., 2015, 5, 25.

15 L. L. Shao, M. Chen, T. Z. Ren and Z. Y. Yuan, J. Power Sources, 2015, 274, 791.

16 K. Zhou, Y. Zhu, X. Yang and C. Li, Electroanalysis, 2011, 23, 862. 
17 L. Kong, X. Lu, X. Bian, W. Zhang and C. Wang, ACS Appl. Mater. Interfaces, 2010, 3, 35.

18 L. M. Cui, Y. G. Wang, L. Gao, L. H. Hu, L. G. Yan, Q. Wei and B. Du, Chem. Eng. J., 2015, 281, 1.

19 Z. B. Lei, Z. H. Liu, H. J. Wang, X. X. Sun, L. Lu and X. S. Zhao, J. Mater. Chem. A, 2013, 1, 2313.

20 X. Y. Zhao, J. P. Tu, Y. Lu, J. B. Cai, Y. J. Zhang, X. L. Wang and C. D. Gu, Electrochim. Acta, 2013, 113, 256.

21 M. Seredych and T. J. Bandosz, J. Mater. Chem. A, 2013, 1, 11717.

22 Y. Corre, M. Seredych and T. J. Bandosz, Carbon, 2013, 55, 176.

23 X. J. He, J. X. Wang, G. H. Xu, M. X. Yu and M. B. Wu, Diamond Relat. Mater., 2016, 66, 119.

24 Y. Ma, C. Ma, J. Sheng, H. X. Zhang, R. R. Wang, Z. Y. Xie and J. L. Shi, J. Colloid Interface Sci., 2016, 461, 96.

25 X. L. Zhang, S. Y. Luo, Q. L. Lin, Y. M. Wang, X. D. Huang and L. Xiong, J. Anal. Appl. Pyrolysis, 2017, 124, 73.

26 X. L. Zhang, C. H. Zhong, Q. L. Lin, S. Y. Luo, X. Q. Zhang and C. Q. Fang, J. Anal. Appl. Pyrolysis, 2017, 126, 154.

27 Z. B. Lei, N. Christov and X. S. Zhao, Energy Environ. Sci., 2011, 4, 1866.

28 Z. M. Wang, W. D. Wang, N. Coombs, N. Soheilnia and G. A. Ozin, ACS Nano, 2010, 4, 7437.

29 S. B. Yang, X. L. Feng, L. Wang, K. Tang, J. Maier and K. Müllen, Angew. Chem., Int. Ed., 2010, 49, 4795.

30 C. W. Lee, K. C. Roh and K. B. Kim, Nanoscale, 2013, 5, 9604. 31 Y. F. Song, J. Yang, K. Wang, S. Haller, Y. G. Wang, C. X. Wang and Y. Y. Xia, Carbon, 2016, 96, 955.

32 R. L. Liu, L. Wan, S. Q. Liu, L. X. Pan, D. Q. Wu and D. Y. Zhao, Adv. Funct. Mater., 2015, 25, 526.

33 X. H. Jin, Q. L. Wei, H. J. Huang, M. X. Wang and Y. M. Huang, Chin. J. Chem., 2012, 30, 2805.

34 D. S. Zhang, X. R. Wen, L. Y. Shi, T. T. Yan and J. P. Zhang, Nanoscale, 2012, 4, 5440.
35 L. Wang, L. Sun, C. G. Tian, T. X. Tan, G. Mu, H. X. Zhang and H. G. Hu, RSC Adv., 2012, 2, 8359.

36 C. J. Guerrero and B. F. Caballero, Mater. Chem. Phys., 2015, 153, 209.

37 M. X. Wang, C. Y. Wang, T. Q. Li and Z. J. Hu, Carbon, 2008, 46, 84.

38 C. J. Guerrero and B. F. Caballero, Mater. Chem. Phys., 2015, 153, 209.

39 Y. L. Cheng, T. H. Li, C. Q. Fang, M. R. Zhang, X. L. Liu, R. E. Yu and J. B. Hu, Appl. Surf. Sci., 2013, 182, 862.

40 S. X. Zhang, Q. Weng, F. Q. Zhao, H. X. Gao, P. Chen, X. B. Chen and Z. W. An, J. Colloid Interface Sci., 2017, 496, 35.

41 Y. F. Wang, H. H. Jiang, S. W. Ye, J. M. Zhou, J. H. Chen, Q. Q. Zeng, H. Yang and T. X. Liang, Funct. Mater. Lett., 2019, 12, 1950042.

42 A. Nabi, S. Tasneem, C. G. Jesudason, V. S. Lee and S. B. Zain, J. Mol. Liq., 2018, 256, 100.

43 Y. L. Cheng, L. Yang, C. Q. Fang and X. Guo, J. Anal. Appl. Pyrolysis, 2016, 122, 387.

44 Y. L. Cheng, Q. L. Zhang, C. Q. Fang, Y. Ouyang, J. Chen, X. Yu and D. H. Liu, J. Anal. Appl. Pyrolysis, 2018, 129, 154.

45 M. Liu, J. Qian, Y. Zhao, D. Zhu, L. Gan and L. Chen, J. Mater. Chem. A, 2015, 3, 11517.

46 D. Wang, Z. Geng, B. Li and C. Zhang, Electrochim. Acta, 2015, 173, 377.

47 Y. L. Cheng, Q. L. Zhang, C. Q. Fang, Z. G. Huang, J. Chen, L. L. Wu and H. T. Wang, J. Electrochem. Soc., 2019, 166, A1. 48 Y. L. Cheng, Q. L. Zhang, C. Q. Fang and S. H. Guo, J. Electrochem. Soc., 2017, 164, A1845.

49 W. Xing, S. Z. Qiao, R. G. Ding, F. Li, G. Q. Lu, Z. F. Yan and H. M. Cheng, Carbon, 2006, 44, 216.

50 C. W. Huang, C. H. Hsu, P. L. Kuo, C. T. Hsieh and H. Teng, Carbon, 2011, 49, 895.

51 L. L. Zhang, S. Y. Zhao, X. N. Tian and X. S. Zhao, Langmuir, 2010, 26, 17624. 